\title{
Action Emulation Between Canonical Models
}

\author{
Floor Sietsma Jan van Eijck
}

May 23, 2013

\begin{abstract}
In this paper we investigate Kripke models, used to model knowledge or belief in a static situation, and action models, used to model communicative actions that change this knowledge or belief. The appropriate notion for structural equivalence between modal structures such as Kripke models is bisimulation: Kripke models that are bisimilar are modally equivalent. We would like to find a structural relation that can play the same role for the action models that play a prominent role in information updating. Two action models are equivalent if they yield the same results when updating Kripke models. More precisely, two action models are equivalent if it holds for all Kripke models that the result of updating with one action model is bisimilar to the result of updating with the other action model. We propose a new notion of action emulation that characterizes the structural equivalence of the important class of canonical action models. Since every action model has an equivalent canonical action model, this gives a method to decide the equivalence of any pair of action models. We also give a partial result that holds for the class of all action models. Our results extend the work in [4].
\end{abstract}

\section{Introduction}

In Dynamic Epistemic Logic (or: DEL), Kripke models are used to model the knowledge of a group of agents in a certain situation, and action models to update these models as a result of information flow and/or factual change. In this paper we address an important technical question concerning these models, namely: when are two action models equivalent? And how can one detect such an equivalence?

Kripke models may be used to interpret any modal logic and they are well studied. In particular, it is well known (see e.g. [2]) that two Kripke models are semantically equivalent if and only if there exists a relation between them that is a bisimulation.

Action models were introduced in [1] as a way to model communicative actions rather than static situations. Two action models are considered equivalent if they have the same effect on all possible Kripke models. The next step would be to find a notion corresponding to bisimulation for action models. In other words, finding an easy way to tell whether two action models are equivalent just by looking at their structure. This paper is dedicated to finding the right definition of a relation between action models called action emulation, such that there exists an action emulation between two action models if and only if they are equivalent. 
The problem we study has been addressed before in [4]. There, a partial solution is provided. A notion of action emulation parametrized by a world from a canonical Kripke model is constructed (henceforth: parametrized action emulation). The union of all these relations is shown to coincide with action model equivalence. This cannot be the final word, for using this notion of action emulation one would have to construct a relation between the action models for every world from a canonical Kripke model, which is tedious work. We would like to improve on this result by giving a direct definition of action emulation between action models. The definition we propose here is simpler than the one from [4] because it does not involve worlds from a canonical Kripke model and is constructed as one single relation, rather than being the union of multiple relations.

This paper is a revised version of [3, Chapter 7]. It is set up as follows. First we give some established definitions related to Kripke models and action models. Then we introduce the class of canonical action models and show that every action model has an equivalent canonical action model. We give a definition of action emulation and show that the existence of an action emulation between two action models implies their equivalence. Then we prove that the converse holds for the class of canonical action models. Because any action model has an equivalent canonical action model, this way any two action models can be compared.

\section{Models and Action Models}

Let $P$ be a countable set of proposition letters an let $A$ be a finite set of labels. The modal language $\mathcal{L}^{M}$ over $P$ and $A$ is given by:

$$
\phi::=p|\neg \phi| \phi \vee \phi \mid \diamond_{a} \phi
$$

where $p$ ranges over $P$ and $a$ over $A$. This is very similar to the language of epistemic logic, only instead of epistemic programs we use a modality, $\diamond_{a} \phi$. It may stand for knowledge, obligation, or any other of a wide range of interpretations.

We will employ the usual shorthands: $\phi \wedge \psi$ for $\neg(\phi \vee \psi), \phi \rightarrow \psi$ for $\neg \phi \vee \psi$, $\phi \leftrightarrow \psi$ for $(\phi \rightarrow \psi) \wedge(\psi \rightarrow \phi)$, and $\square_{a} \phi$ for $\left.\neg \vee_{a} \neg \phi\right)$. The modality $\square_{a} \phi$ is the dual of $\nabla_{a} \phi$.

We will interpret the formulas from $\mathcal{L}^{M}$ on Kripke models. They are defined as follows:

Definition $1 A$ Kripke model $\mathcal{M}$ over a label set $A$ and an proposition set $P$ is a triple $\left(W, \mathrm{Val}, R, W_{0}\right)$, where $W$ is a non-empty set of worlds, Val is a function that assigns to every world $w \in W$ a set $\operatorname{Val}(w) \subseteq P, R$ is a function that assigns to every label $a \in A$ a binary relation $R_{a} \subseteq W^{2}$ and $W_{0}$ is the set of actual worlds. We will sometimes use $W_{\mathcal{M}}, V l_{\mathcal{M}}, R_{\mathcal{M}}$ to denote the elements of the Kripke model $\mathcal{M}$.

In epistemic logic it is common to view the $R_{a}$ as agent accessibilities, but we will not insist on this in the present context. Our results also apply if the modalities $\nabla_{a}$ and $\square_{a}$ do not represent the knowledge of an agent. In particular, we will not assume that the $R_{a}$ are equivalence relations. As an alternate notation for $R_{a}$, we will use $\stackrel{a}{\rightarrow}$. 
The semantics of $\mathcal{L}^{M}$ is given by the following truth definition in a model $\mathcal{M}=\left(W, \mathrm{Val}, R, W_{0}\right)$ :

\section{Definition 2 (Truth)}

$$
\begin{array}{rll}
\mathcal{M}=_{w} p & \text { iff } & p \in \operatorname{Val}(w) \\
\mathcal{M} \models_{w} \neg \phi & \text { iff } & \mathcal{M} \models_{w} \phi \\
\mathcal{M} \models_{w} \phi_{1} \vee \phi_{2} & \text { iff } & \mathcal{M}={ }_{w} \phi_{1} \text { or } \mathcal{M}=_{w} \phi_{2} \\
\mathcal{M}=_{w} \nabla_{a} \phi & \text { iff } & \exists w^{\prime} \in W: w \stackrel{a}{\rightarrow} w^{\prime} \text { and } w^{\prime} \models \phi .
\end{array}
$$

The semantics of the modality $\nabla_{a}$ is straightforward: $\nabla_{a} \phi$ holds if it is possible to do an $a$-step to a world where $\phi$ holds. Dually, $\square_{a} \phi$ holds if every world that is reachable with an $a$-step satisfies $\phi$.

A Kripke model is a description of a certain situation. When the situation changes, the Kripke model needs to be updated. For this purpose one can use action models. An action model is like a Kripke model, only instead of possible worlds it has possible events which have a formula called a precondition instead of a valuation. Also, we will assume that the event set of an action model is finite.

Formally, an action model is defined as follows:

Definition 3 An action model over a label set $A$ and an proposition set $P$ isis a tuple $\mathcal{A}=\left(E\right.$, Pre $\left., R, E_{0}\right)$ where $E$ is a non-empty finite set of events, Pre is a function that assigns to each event in $E$ an $\mathcal{L}^{M}$-formula over $P$ (its precondition), $R$ is a function that assigns to every label $a \in A$ a binary relation $R_{a} \subseteq E^{2}$ and $E_{0} \subseteq E$ is the set of actual events. We will sometimes use $E_{\mathcal{A}}, \operatorname{Pre}_{\mathcal{A}}, R_{\mathcal{A}}$ to denote the elements of the action model.

When a Kripke model is updated with an action model, every world from the Kripke model gets matched with every event from the action model, provided that the world satisfies the precondition of the event. This operation is called the product update. If there is a relation between two worlds in the Kripke model and these worlds are matched with two events in the action model, then the relation is only preserved if there is also a relation between the two events in the action model. The formal definition of the product update is as follows:

Definition 4 (Update) Given a Kripke model $\mathcal{M}=\left(W\right.$, Val $\left., R, W_{0}\right)$ and an action model $\mathcal{A}=\left(E\right.$, Pre, $\left.S, E_{0}\right)$, the result of updating $\mathcal{M}$ with $\mathcal{A}$ is the model $\mathcal{M} \otimes \mathcal{A}=\left(W^{\prime}, V_{a l}^{\prime}, R^{\prime}, W_{0}^{\prime}\right)$ given by

$$
\begin{aligned}
W^{\prime} & :=\left\{(w, e) \mid w \in W, e \in E, \mathcal{M} \models_{w} \operatorname{Pre}(e)\right\} \\
(w, d) R_{a}^{\prime}(v, e) & \text { iff } \quad w R_{a} v \text { and } d S_{a} e \\
\operatorname{Val}^{\prime}((w, e)) & :=\operatorname{Val}(w), \\
W_{0}^{\prime} & :=\left\{(w, e) \in W^{\prime} \mid w \in W_{0} \text { and } e \in E_{0}\right\}
\end{aligned}
$$

Action models model events that change the situation that is modeled by a Kripke model. For example, in an epistemic interpretation of the Kripke model, an action model can be seen as a communicative event changing the knowledge of the agents. 


\section{Canonical Models}

Given a formula $\varphi$, we define its single negation as follows: if $\phi$ is of the form $\neg \psi$, then $\sim \phi=\psi$, and otherwise $\sim \phi=\neg \phi$. We will implicitly use the equivalences of $\neg \square_{a} \phi$ and $\nabla_{a} \sim \phi, \neg \vee_{a} \phi$ and $\square_{a} \sim \phi, \neg(\phi \wedge \psi)$ and $\sim \phi \vee \sim \psi$ and $\neg(\phi \vee \psi)$ and $\sim \phi \wedge \sim \psi$.

The definition of single negation allows us to define the closure of a formula or a set of formulas.

Definition 5 Given a formula $\phi$, we define its closure $C(\phi)$ as the smallest set containing $\phi$ that is closed under taking subformulas and single negations. Given a finite set of formulas $\Phi$, we define $C(\Phi):=\bigcup_{\phi \in \Phi} C(\phi)$.

The following example shows how this definition works out.

Example $1 p \wedge \diamond_{a} \neg p$ has the following closure:

$$
C\left(p \wedge \diamond_{a} \neg p\right)=\left\{p \wedge \diamond_{a} \neg p, \neg p \vee \square_{a} p, p, \neg p, \diamond_{a} \neg p, \square_{a} p\right\} .
$$

Definition 6 An atom over a finite set of formulas $\Phi$ is a maximal consistent subset of $C(\Phi)$.

An atom over $\Phi$ can be seen as a complete description of a possible state of the world, if one only considers the formulas in $\Phi$. We will use these atoms to construct canonical models.

Example $2\left\{p \wedge \diamond_{a} \neg p\right\}$ has four atoms:

- $\left\{p \wedge \diamond_{a} \neg p, p, \diamond_{a} \neg p\right\}$,

- $\left\{\neg p \vee \square_{a} p, \neg p, \diamond_{a} \neg p\right\}$,

- $\left\{\neg p \vee \square_{a} p, p, \square_{a} p\right\}$,

- $\left\{\neg p \vee \square_{a} p, \neg p, \square_{a} p\right\}$.

A canonical Kripke model is a model that has a world for every possible atom over a certain set of formulas. Therefore it models all possible truth values of these formulas and their subformulas.

Definition 7 Let $\Phi$ is a finite set of formulas, let $\Sigma$ be the set of atoms over $\Phi$ and let $\left\{p_{\sigma} \mid \sigma \in \Sigma\right\}$ be a set of unique propositions not occurring in formulas in $\Phi$. Then the canonical Kripke model $\mathcal{M}^{c}=\left(W^{c}, \operatorname{Val}^{c}, R^{c}, W_{0}^{c}\right)$ over $\Phi$ is defined as

$$
\begin{aligned}
& W^{c} \quad:=\Sigma \\
& \operatorname{Val}^{c}(\sigma):=(P \cap \sigma) \cup\left\{p_{\sigma}\right\} \\
& \sigma \stackrel{a}{\rightarrow} \sigma^{\prime} \quad \text { iff } \quad \wedge \sigma \wedge \nabla_{a} \wedge \sigma^{\prime} \text { is consistent } \\
& W_{0}^{c} \quad:=\Sigma
\end{aligned}
$$

Note that every world in the canonical model has a unique valuation, due to the propositions $\left\{p_{\sigma} \mid \sigma \in \Sigma\right\}$.

Every world in the canonical model corresponds to an atom, and there is an $a$-relation from one atom to another if the formulas in the first atom are consistent with $\nabla_{a} \phi$, for any formula $\phi$ in the second atom. The following is shown in $[2]$. 
Theorem 3 Let $\mathcal{M}^{c}$ be the canonical model over a set of formulas $\Phi$. Then for any atom $\sigma$ over $\Phi$ and for any formula $\phi \in C(\Phi)$,

$$
\mathcal{M}^{c}=_{\sigma} \phi \text { iff } \phi \in \sigma \text {. }
$$

\section{Action Models and Bisimulation}

Two Kripke models are considered equivalent when they are bisimilar. If they are bisimilar, they satisfy exactly the same modal formulas. They can be viewed as two different representations of the exact same situation. For the record:

Definition 8 (Bisimulation) Let $M, N$ be Kripke models. A relation $Z \subseteq$ $W_{M} \times W_{N}$ is a bisimulation if for all $w \in W_{M}$ and $v \in W_{N}$ such that $w Z v$ the following hold:

Invariance $\operatorname{Val}_{M}(w)=\operatorname{Val}_{N}(v)$,

Zig for all $a \in A$ and all worlds $w^{\prime} \in W_{M}$ with $w \stackrel{a}{\rightarrow} w^{\prime}$ there is a world $v^{\prime} \in W_{N}$ with $v \stackrel{a}{\rightarrow} v^{\prime}$ and $w^{\prime} Z v^{\prime}$

Zag for all $a \in A$ and all worlds $v^{\prime} \in W_{N}$ with $v \stackrel{a}{\rightarrow} v^{\prime}$ there is a world $w^{\prime} \in W_{M}$ with $w \stackrel{a}{\rightarrow} w^{\prime}$ and $w^{\prime} Z v^{\prime}$.

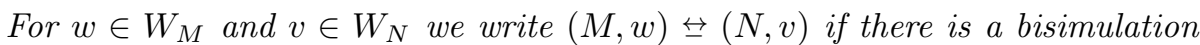
between $M$ and $N$ connecting $w$ and $v$.

We write $M \leftrightarrow N$ and say that $M$ and $N$ are bisimilar if there is a bisimulation connecting every point in $W_{M}$ with some point in $W_{N}$ and vice versa.

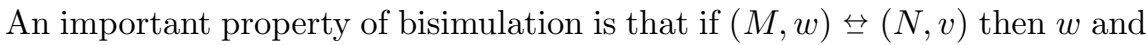
$v$ satisfy the same formulas of $L$ (for a proof, see [2]).

Action models model a communicative event. Just like Kripke models, sometimes two different action models model the same thing. In the case of action models, this means they model the same communicative event. This is signified by the fact that they have the same effect on all Kripke models. That is, if we apply the two different action models on the same Kripke model, the resulting models will be bisimilar.

Definition 9 We will say that two action models $\mathcal{A}$ and $\mathcal{B}$ are equivalent, notation $\mathcal{A} \equiv \mathcal{B}$, if for any Kripke model $\mathcal{M}$,

$$
\mathcal{M} \otimes \mathcal{A} \leftrightarrow \mathcal{M} \otimes \mathcal{B}
$$

Note that if two action models are equivalent, then the result of updating a Kripke model with one of them is bisimilar to the result of the update with the other, even if the model mentions propositions that are not mentioned in the action models.

The problem we face in this paper is to find a structural relation between action models that signifies their equivalence, just like bisimulation does for Kripke models. When two action models $\mathcal{A}$ and $\mathcal{B}$ are equivalent, every world that matches an event of $\mathcal{A}$ should also match an event of $\mathcal{B}$ and vice versa. Furthermore, the results of these matchings should be bisimilar. 
The first solution that comes to mind is to apply bisimulation to action models. One could replace the requirement that the worlds have the same valuation with the requirement that their preconditions are semantically equivalent. This gives the following definition:

Definition 10 Two action models $\mathcal{A}$ and $\mathcal{B}$ are bisimilar if there is a relation $Z: E^{\mathcal{A}} \times E^{\mathcal{B}}$ which is total on $E_{0}^{\mathcal{A}} \times E_{0}^{\mathcal{B}}$, such that the following conditions hold for any $x, y$ such that $x Z y$ :

Invariance $\operatorname{Pre}^{\mathcal{A}}(x) \equiv \operatorname{Pre}^{\mathcal{B}}(y)$,

Zig for any label $a \in A$, if there is a world $x^{\prime}$ such that $x \stackrel{a^{\mathcal{A}}}{\rightarrow} x^{\prime}$ then there must be a world $y^{\prime}$ such that $y \stackrel{a}{\rightarrow} y^{\prime}$ and $\left(x^{\prime}, y^{\prime}\right) \in Z$,

Zag for any label $a \in A$, if there is a world $y^{\prime}$ such that $y \stackrel{a}{\rightarrow} y^{\prime}$ then there must be a world $x^{\prime}$ such that $x \stackrel{a}{\rightarrow} x^{\prime}$ and $\left(x^{\prime}, y^{\prime}\right) \in Z$.

Here $\equiv$ signifies logical equivalence.

However, this bisimulation for action models does not have the required properties. The following example, which is inspired by [4], shows why not.

Example 4 Consider the following two action models, where all relations are symmetric and reflexive relations are present for all events but not drawn in the picture. Gray shading indicates the actual event.
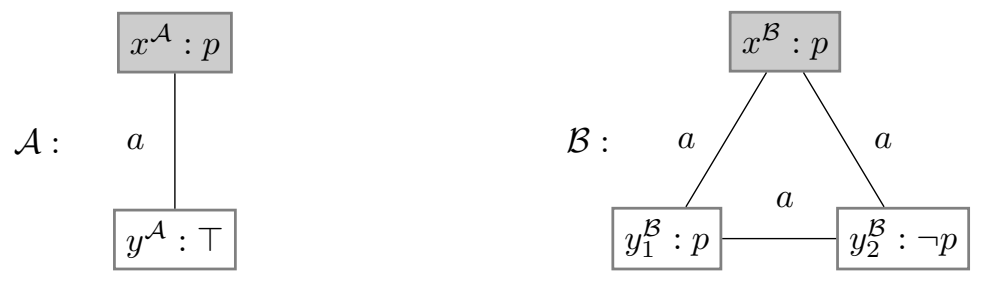

These two models are not bisimilar: there is no event in $\mathcal{B}$ that has a precondition which is logically equivalent to the precondition of $y^{\mathcal{A}}$ in $\mathcal{A}$. Therefore the $a$-step from the actual event $x^{\mathcal{A}}$ to $y^{\mathcal{A}}$ cannot be matched by an $a$-step from $x^{\mathcal{B}}$ to an event that is bisimilar to $y^{\mathcal{A}}$.

However, they are equivalent. One can see this as follows. Clearly any world that matches event $x^{\mathcal{A}}$ in $\mathcal{A}$ will match event $x^{\mathcal{B}}$ in $\mathcal{B}$ and vice versa. Furthermore, any world that matches event $y^{\mathcal{A}}$ in $\mathcal{A}$ will match $y_{1}^{\mathcal{B}}$ in $\mathcal{B}$ if it satisfies $p$, and $y_{2}^{\mathcal{B}}$ in $\mathcal{B}$ if it does not satisfy $p$. Since the relations between $x^{\mathcal{B}}$ and $y_{1}^{\mathcal{B}}$ and $y_{2}^{\mathcal{B}}$ in $\mathcal{B}$ are the same as the relations between $x^{\mathcal{A}}$ and $y^{\mathcal{A}}$ in $\mathcal{A}$, the results of these matchings are bisimilar.

More formally, if $\mathcal{M}$ is a Kripke model then we define the relation $Z$ on $W^{\mathcal{M} \otimes \mathcal{A}} \times W^{\mathcal{M} \otimes \mathcal{B}}$ as follows. For any $w \in W^{\mathcal{M}}$,

$$
\begin{array}{lr}
\left(w, x^{\mathcal{A}}\right) Z\left(w, x^{\mathcal{B}}\right), & \\
\left(w, y^{\mathcal{A}}\right) Z\left(w, y_{1}^{\mathcal{B}}\right) & \text { if } w=p \\
\left(w, y^{\mathcal{A}}\right) Z\left(w, y_{2}^{\mathcal{B}}\right) & \text { otherwise. }
\end{array}
$$

It is not hard to check that $Z$ is indeed a bisimulation between $\mathcal{M} \otimes \mathcal{A}$ and $\mathcal{M} \otimes \mathcal{B}$. 
The above example shows that the problem of detecting equivalence between action models is not solved by simply adapting the definition of bisimulation. Therefore we would like to find a more sophisticated relation between action models. We will define such a relation later in this paper, but first we will show that there is a way to detect action model equivalence by looking at canonical Kripke models.

Definition 11 Given an action model $\mathcal{A}$, we define its language $\Lambda_{\mathcal{A}}$ as the closure of the union of the preconditions of all its events.

We start with a useful lemma.

Lemma 1 Take two action models $\mathcal{A}$ and $\mathcal{B}$ such that $\Phi=\Lambda_{\mathcal{A}} \cup \Lambda_{\mathcal{B}}$ and let $\mathcal{M}^{c}$ be the canonical Kripke model over $\Phi$. Let $w, v$ be atoms of $\mathcal{M}^{c}$. Then $\operatorname{Val}^{c}(w)=\operatorname{Val}^{c}(v)$ implies $w=v$.

Proof. Every world in $\mathcal{M}^{c}$ has a unique valuation by the definition of a canonical Kripke model.

In [4], the following useful observation is made about canonical Kripke models and action model equivalence. We rephrase the proof here.

Theorem 5 Take two action models $\mathcal{A}$ and $\mathcal{B}$ such that $\Phi=\Lambda_{\mathcal{A}} \cup \Lambda_{\mathcal{B}}$ and let $\mathcal{M}^{c}$ be the canonical Kripke model over $\Phi$. Then the following holds:

$$
\mathcal{A} \equiv \mathcal{B} \text { iff } \mathcal{M}^{c} \otimes \mathcal{A} \leftrightarrow \mathcal{M}^{c} \otimes \mathcal{B}
$$

ProOF. The proof for the left to right direction is immediate by the definition of action model equivalence. For the right to left direction, suppose $\mathcal{M}^{c} \otimes \mathcal{A} \leftrightarrow$ $\mathcal{M}^{c} \otimes \mathcal{B}$. Then there is a bisimulation $Z: W^{\mathcal{M}^{c} \otimes \mathcal{A}} \times W^{\mathcal{M}^{c} \otimes \mathcal{B}}$. Since the valuation of $\mathcal{M}^{c}$ is preserved in $\mathcal{M}^{c} \otimes \mathcal{A}$ and $\mathcal{M}^{c} \otimes \mathcal{B}$, by Lemma 1 we have for any $(w, x) \in \mathcal{M}^{c} \otimes \mathcal{A}$ and $(v, y) \in \mathcal{M}^{c} \otimes \mathcal{B}$ that $(w, x) Z(v, y)$ implies $w=v$.

Take any Kripke model $\mathcal{M}$. For any world $w \in W^{\mathcal{M}}$, let $\sigma_{w}$ be the set of formulas in $C(\Phi)$ that are satisfied in $w$. Observe that $\sigma_{w}$ is an atom: for every $\phi$ that is in $C(\Phi)$, either $\phi$ or $\sim \phi$ is satisfied in $w$. The union of all such formulas is a maximal consistent subset of $C(\Phi)$. Define a relation $Y: W^{\mathcal{M} \otimes \mathcal{A}} \times W^{\mathcal{M} \otimes \mathcal{B}}$ as follows:

$$
(w, x) Y(v, y) \text { iff } w=v \text { and }\left(\sigma_{w}, x\right) Z\left(\sigma_{w}, y\right) .
$$

We will show that $Y$ is a bisimulation.

Suppose $(w, x) Y(w, y)$. Then $\left(\sigma_{w}, x\right) Z\left(\sigma_{w}, y\right)$.

To see that Invariance is satisfied, observe that the valuations of $(w, x)$ and of $(w, y)$ are both inherited from $w$ and therefore identical.

For Zig, suppose $(w, x) \stackrel{a}{\rightarrow}\left(w^{\prime}, x^{\prime}\right)$. Because $\operatorname{Pre}(x) \in \Lambda_{A}, \operatorname{Pre}(x) \in \Phi$. Because $\mathcal{M} \models_{w} \operatorname{Pre}(x)$, then we have $\operatorname{Pre}(x) \in \sigma_{w}$. By a similar reasoning, $\operatorname{Pre}\left(x^{\prime}\right) \in \sigma_{w^{\prime}}$. Therefore, $\left(\sigma_{w}, x\right)$ and $\left(\sigma_{w^{\prime}}, x^{\prime}\right)$ are in the domain of $\mathcal{M}^{c} \otimes \mathcal{A}$. From $(w, x) \stackrel{a}{\rightarrow}\left(w^{\prime}, x^{\prime}\right)$ we infer that $w \stackrel{a}{\rightarrow} w^{\prime}$ and $x \stackrel{a}{\rightarrow} x^{\prime}$. Because $\mathcal{M} \models w \wedge \sigma_{w}$ and $\mathcal{M}==_{w^{\prime}} \wedge \sigma_{w^{\prime}}$, then $\bigwedge \sigma_{w} \wedge \diamond_{a}\left(\bigwedge \sigma_{w^{\prime}}\right)$ is consistent, so $\sigma_{w} \stackrel{a}{\rightarrow} \sigma_{w^{\prime}}$. This implies that $\left(\sigma_{w}, x\right) \stackrel{a}{\rightarrow}\left(\sigma_{w^{\prime}}, x^{\prime}\right)$. Because $\left(\sigma_{w}, x\right) Z\left(\sigma_{w}, y\right)$, there must be $\left(v, y^{\prime}\right)$ such that $\left(\sigma_{w}, y\right) \stackrel{a}{\rightarrow}\left(v, y^{\prime}\right)$ and $\left(\sigma_{w^{\prime}}, x^{\prime}\right) Z\left(v, y^{\prime}\right)$. By Lemma $1\left(\sigma_{w^{\prime}}, x^{\prime}\right) Z\left(v, y^{\prime}\right)$ 
implies that $v=\sigma_{w^{\prime}}$, so $\left(\sigma_{w^{\prime}}, x^{\prime}\right) Z\left(\sigma_{w^{\prime}}, y^{\prime}\right)$. Then $\operatorname{Pre}\left(y^{\prime}\right) \in \sigma_{w^{\prime}}$ so $\mathcal{M} \models_{w^{\prime}}$ $\operatorname{Pre}\left(y^{\prime}\right)$. Then $\left(w^{\prime}, y^{\prime}\right)$ is in the domain of $\mathcal{M} \otimes \mathcal{B}$ and $\left(w^{\prime}, x^{\prime}\right) Y\left(w^{\prime}, y^{\prime}\right)$. Since $\left(\sigma_{w}, y\right) \stackrel{a}{\rightarrow}\left(\sigma_{w^{\prime}}, y^{\prime}\right)$ it holds that $y \stackrel{a}{\rightarrow} y^{\prime}$. Since we already knew that $w \stackrel{a}{\rightarrow} w^{\prime}$, this shows that $(w, y) \stackrel{a}{\rightarrow}\left(w^{\prime}, y^{\prime}\right)$.

The proof for Zag is analogous.

To see that $Y$ is total, take some $(w, x) \in W_{0}^{\mathcal{M} \otimes \mathcal{A}}$. Then $\mathcal{M} \models_{w} \operatorname{Pre}(x)$ so $\operatorname{Pre}(x) \in \sigma_{w}$. Then $\mathcal{M}^{c} \models_{\sigma_{w}} \operatorname{Pre}(x)$, so $\left(\sigma_{w}, x\right) \in \mathcal{M}^{c} \otimes \mathcal{A}$. Then there is some $\left(\sigma_{v}, y\right)$ such that $\left(\sigma_{w}, x\right) Z\left(\sigma_{v}, y\right)$. By Lemma 1 this implies that $\sigma_{w}=\sigma_{v}$, so $\left(\sigma_{w}, x\right) Z\left(\sigma_{w}, y\right)$. So $\mathcal{M}^{c} \models_{\sigma_{w}} \operatorname{Pre}(y)$, and then $\operatorname{Pre}(y) \in \sigma_{w}$ so $\mathcal{M} \models_{w} \operatorname{Pre}(y)$ and $(w, y)$ is in the domain of $\mathcal{M} \otimes \mathcal{B}$. So $(w, x) Y(w, y)$. The proof for totality in the other direction is analogous.

This theorem demonstrates a straightforward procedure to check whether two action models are equivalent: simply construct the canonical Kripke model for the set of formulas consisting of their preconditions, and see whether the update results on this model bisimulate. Even though this is not complicated, it is a very inefficient method: the size of the canonical Kripke model is exponential in the number of subformulas of the preconditions.

\section{Parametrized Action Emulation}

Theorem 5 is the motivation, in [4], to construct a relation which is parametrized by worlds in the canonical Kripke model. This parametrized action emulation does not yet lead to an efficient method for deciding action model equivalence, because every world in the canonical Kripke model has to be computed. However, we take it as a starting point for further investigations. It is defined as follows.

Definition 12 Given two action models $\mathcal{A}$ and $\mathcal{B}$, let $\Sigma$ be the set of atoms over $\Lambda_{\mathcal{A}} \cup \Lambda_{\mathcal{B}}$. Given some $x \in E^{\mathcal{A}} \cup E^{\mathcal{B}}$, let $S(x)=\{\sigma \in \Sigma \mid \operatorname{Pre}(x) \in \sigma\}$. A parametrized action emulation between $\mathcal{A}$ and $\mathcal{B}$ is a set of indexed relations $\left\{E_{\sigma}\right\}_{\sigma \in \Sigma}$ such that whenever $x E_{\sigma} y$ the following conditions hold:

Invariance $\operatorname{Pre}(x) \in \sigma$ and $\operatorname{Pre}(y) \in \sigma$.

Zig If $x \stackrel{a}{\rightarrow} x^{\prime}$ then for any $\sigma^{\prime} \in S\left(x^{\prime}\right)$ such that $\sigma \stackrel{a}{\rightarrow} \sigma^{\prime}$ there is $y^{\prime} \in E^{\mathcal{B}}$ with $y \stackrel{a}{\rightarrow} y^{\prime}$ and $x^{\prime} E_{\sigma^{\prime}} y^{\prime}$. In a picture:

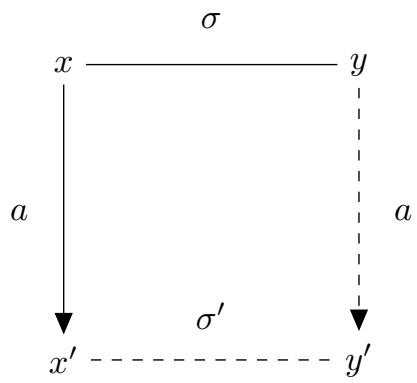

Zag If $y \stackrel{a}{\rightarrow} y^{\prime}$ then for any $\sigma^{\prime} \in S\left(y^{\prime}\right)$ such that $\sigma \stackrel{a}{\rightarrow} \sigma^{\prime}$ there is $x^{\prime} \in E^{\mathcal{A}}$ with $x \stackrel{a}{\rightarrow} x^{\prime}$ and $x^{\prime} E_{\sigma^{\prime}} y^{\prime}$. In a picture: 


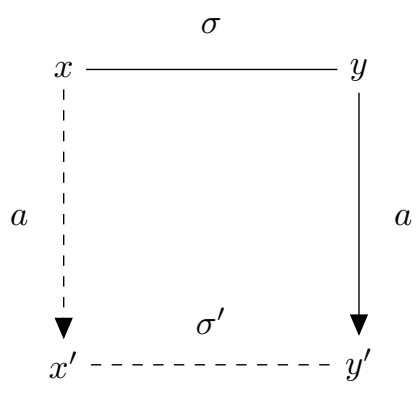

We say that $\mathcal{A}$ and $\mathcal{B}$ emulate parametrized by the canonical model if for every $x \in E_{0}^{\mathcal{A}}$ and for every $\sigma \in S(x)$ there is $y \in E_{0}^{\mathcal{B}}$ with $x E_{\sigma} y$, and vice versa. Notation: $\mathcal{A} \leftrightarrows{ }_{S} \mathcal{B}$

It is shown in [4] that this relation indeed characterizes action model equivalence:

Theorem 6 For any two action models $\mathcal{A}$ and $\mathcal{B}$,

$$
\mathcal{A} \equiv \mathcal{B} \text { iff } \mathcal{A} \leftrightarrows_{S} \mathcal{B}
$$

To see why this definition works, observe that any world $w$ from any Kripke model $\mathcal{M}$ has a corresponding atom $w^{*}$. Then if $\mathcal{A} \leftrightarrows_{S} \mathcal{B}$, there must be for every $x \in E_{\mathcal{A}}$ such that $\mathcal{M} \models_{w} \operatorname{Pre}(x)$ some event $y \in E_{\mathcal{B}}$ such that $x E_{w^{*}} y$. Then $\mathcal{M}={ }_{w} \operatorname{Pre}(y)$, and it is not hard to show that $(w, x)$ is bisimilar to $(w, y)$.

\section{Non-parametrized Action Emulation}

Rather than constructing the canonical Kripke model, we would like to find a definition of a direct relation between action models that signifies their equivalence.

However, Definition 12 leaves us with the same problem as before: it requires the computation of a large number of atoms. One even has to compute a separate relation for every possible atom. We want to improve on this by finding a non-parametrized notion of action emulation.

Checking whether two action models are equivalent is complicated because one world from a Kripke model may match multiple events in the action model and one event in the action model may match multiple worlds in the Kripke model. Moreover, usually there is no direct mapping between $\mathcal{A}$ and $\mathcal{B}$ such that an event in $\mathcal{A}$ matches the exact same worlds in the Kripke model as the related event in $\mathcal{B}$. To circumvent these complications we consider canonical action models.

Definition 13 An action model $\mathcal{A}$ is canonical over a finite set of $\mathcal{L}^{M}$ formulas $\Phi$ if every precondition is the conjunction of an atom over $\Phi$ and for every $x, x^{\prime} \in E_{A}$ such that $x \stackrel{a}{\rightarrow} x^{\prime}, \operatorname{Pre}(x) \wedge \nabla_{a} \operatorname{Pre}\left(x^{\prime}\right)$ is consistent.

Note the difference between canonical Kripke models and canonical action models: a canonical Kripke model has a world for every possible atom, and a relation between two worlds if and only if this relation is consistent with the 
contents of the atoms. On the other hand, a canonical action model may be incomplete in the sense that there may be some atom that is not represented as the precondition of an event in the model. Also, a relation between two events may not be present even though it would be consistent with the preconditions of the events.

Example 7 Consider the following action model (reflexive relations present but omitted in the picture):

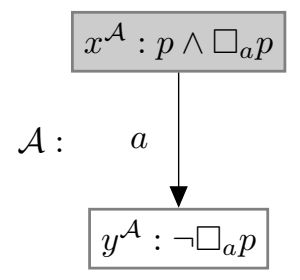

This action model is not canonical over any set of formulas. The reason for this is that the precondition of world $y^{\mathcal{A}}$ is not the conjunction of an atom over the set of formulas $\left\{p, \square_{a} p\right\}$. It is not even an atom over the set of formulas $\left\{\square_{a} p\right\}$, because $p$ is a subformula of $\square_{a} p$.

On the other hand, in the following action model all preconditions are conjunctions of atoms over $\left\{p, \square_{a} p\right\}$ :

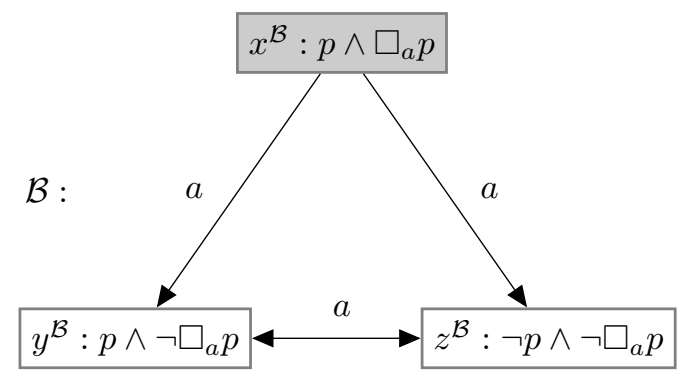

However, this model is still not canonical because there is an $a$-relation from $x^{\mathcal{B}}$ to $z^{\mathcal{B}}$, even though $p \wedge \square_{a} p \wedge \diamond_{a}\left(\neg p \wedge \neg \square_{a} p\right)$ is inconsistent.

The following model does not have any of these inconsistent relations:

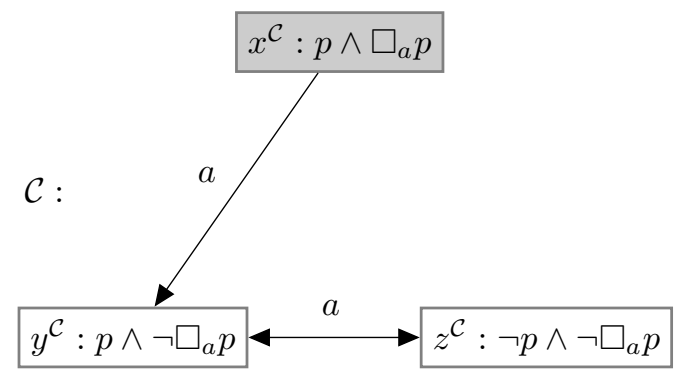

This model is canonical. All its preconditions are conjunctions of atoms over $\left\{p, \square_{a} p\right\}$ and all its relations are consistent. Note that not all atoms are represented in the model: $\neg p \wedge \square_{a} p$ is not present. Also, not all consistent relations are present: for example, there is no relation from $y^{\mathcal{C}}$ to $x^{\mathcal{C}}$, even though this would be allowed. 
The nice thing about canonical action models is that each event completely determines the truth value of all formulas in $\Phi$. In this section we will construct a notion of action emulation that corresponds to action model equivalence for canonical action models. But first we show that every action model has an equivalent canonical action model.

Theorem 8 Every action model has an equivalent canonical action model.

Proof. Take an action model $\mathcal{A}=\left(E\right.$, Pre, $\left.R, E_{0}\right)$. Let $\Sigma$ be the set of atoms over $\Lambda_{\mathcal{A}}$. We construct a new action model $\mathcal{A}^{c}=\left(E^{c}, \operatorname{Pre}^{c}, R^{c}, E_{0}^{c}\right)$ as follows:

$$
\begin{array}{llll}
E^{c} & := & \{(x, \sigma) \mid x \in E, \sigma \in \Sigma, \operatorname{Pre}(x) \in \sigma\}, \\
\operatorname{Pre}^{c}(x, \sigma) & := & \bigwedge \sigma, \\
(x, \sigma) \stackrel{a}{\rightarrow}\left(x^{\prime}, \sigma^{\prime}\right) & \text { iff } & x \stackrel{a}{\rightarrow} x^{\prime} \text { and } \bigwedge \sigma \wedge \diamond_{a} \wedge \sigma^{\prime} \text { is consistent, } \\
E_{0}^{c} & := & \left\{(x, \sigma) \in E^{c} \mid x \in E_{0}\right\} .
\end{array}
$$

It follows from this definition that $\mathcal{A}^{c}$ is canonical. We claim that $\mathcal{A} \equiv \mathcal{A}^{c}$.

Take some model $\mathcal{M}$. Define a relation $Z$ on $\mathcal{M} \otimes \mathcal{A} \times \mathcal{M} \otimes \mathcal{A}^{c}$ as follows:

$$
(w, x) Z(v,(y, \sigma)) \text { iff } w=v \text { and } x=y .
$$

We will start out by showing that $Z$ is total. Take some $(w, x) \in W_{\mathcal{M} \otimes \mathcal{A}}$. Let $\sigma=\left\{\varphi \in \Lambda_{\mathcal{A}} \mid \mathcal{M} \models_{w} \varphi\right\}$. Then $\sigma \in \Sigma$ and $\operatorname{Pre}(x) \in \sigma$ so $(x, \sigma) \in E^{c}$. Clearly, $\mathcal{M} \models_{w} \wedge \sigma$ so $(w,(x, \sigma)) \in W_{\mathcal{M} \otimes \mathcal{A}^{c}}$ and $(w, x) Z(w,(x, \sigma))$. Now take

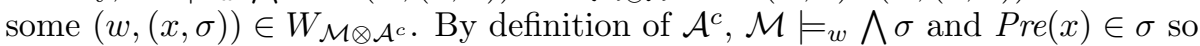
$\mathcal{M}=_{w} \operatorname{Pre}(x)$ and $(w, x) Z(w,(x, \sigma))$.

Now we will show that $Z$ is a bisimulation. Suppose $(w, x) Z(w,(x, \sigma))$. Invariance is satisfied because both $(w, x)$ and $(w,(x, \sigma))$ inherit their valuation from $w$. For Zig, suppose $(w, x) \stackrel{a}{\rightarrow}\left(w^{\prime}, x^{\prime}\right)$. Let $\sigma^{\prime}=\left\{\varphi \in \Lambda_{\mathcal{A}} \mid \mathcal{M} \models w_{w^{\prime}} \varphi\right\}$. By definition of $Z, \mathcal{M}={ }_{w} \wedge \sigma$ and clearly $\mathcal{M}=_{w^{\prime}} \wedge \sigma^{\prime}$ so $\wedge \sigma \wedge \diamond_{a} \wedge \sigma^{\prime}$ is consistent. Then by definition of $R^{c}$ we have $(x, \sigma) \stackrel{a}{\rightarrow}\left(x^{\prime}, \sigma^{\prime}\right)$ so $(w,(x, \sigma)) \stackrel{a}{\rightarrow}$ $\left(w^{\prime},\left(x^{\prime}, \sigma^{\prime}\right)\right)$. Furthermore, $\left(w^{\prime}, x^{\prime}\right) Z\left(w^{\prime},\left(x^{\prime}, \sigma^{\prime}\right)\right)$. This shows satisfaction of Zig.

For Zag, suppose $(w,(x, \sigma)) \stackrel{a}{\rightarrow}\left(w^{\prime},\left(x^{\prime}, \sigma^{\prime}\right)\right)$. Then $w \stackrel{a}{\rightarrow} w^{\prime}$ and $x \stackrel{a}{\rightarrow} x^{\prime}$ so $(w, x) \stackrel{a}{\rightarrow}\left(w^{\prime}, x^{\prime}\right)$. Furthermore, $\left(w^{\prime}, x^{\prime}\right) Z\left(w^{\prime},\left(x^{\prime}, \sigma^{\prime}\right)\right)$. This shows the satisfaction of Zag.

So for every world in the original model, we construct the possible atoms corresponding to that world. We preserve only the relations from the original model that are consistent. This way we construct an equivalent canonical action model. Note that in the previous example, the action model $\mathcal{C}$ would be the result of constructing equivalent canonical models for $\mathcal{A}$ and $\mathcal{B}$ in this manner.

Now we will define a new notion of action emulation, using some abbreviations introduced in [4]:

- If $\stackrel{a}{\rightarrow}$ is a relation on $X \times Z, x \in X$ and $Y \subseteq Z$ then we write $x \stackrel{\bar{a}}{\rightarrow} Y$ to mean that $x \stackrel{a}{\rightarrow} y$ for every $y \in Y$,

- If $E$ is a relation on $X \times Z, x \in X$ and $Y \subseteq Z$ then we write $x \bar{E} Y$ to mean that $x E y$ for every $y \in Y$,

- If $E$ is a relation on $Z \times Y, X \subseteq Z$ and $y \in Y$ then we write $X \bar{E} y$ to mean that $x E y$ for every $x \in X$. 
Definition 14 Given two action models $\mathcal{A}$ and $\mathcal{B}$, a relation $E: E^{\mathcal{A}} \times E^{\mathcal{B}}$ is an action emulation if for any $x \in E^{\mathcal{A}}, y \in E^{\mathcal{B}}$ such that $x E y$ the following hold:

Consistency $\operatorname{Pre}(x) \wedge \operatorname{Pre}(y)$ is consistent.

Zig If $x \stackrel{a}{\rightarrow} x^{\prime}$ then there is $Y^{\prime} \subseteq E_{\mathcal{B}}$ such that $y \stackrel{\bar{a}}{\rightarrow} Y^{\prime}, x^{\prime} \bar{E} Y^{\prime}$ and

$$
\operatorname{Pre}(x) \wedge \operatorname{Pre}(y) \models \square_{a}\left(\operatorname{Pre}\left(x^{\prime}\right) \rightarrow \bigvee_{y^{\prime} \in Y^{\prime}} \operatorname{Pre}\left(y^{\prime}\right)\right)
$$

In a picture:

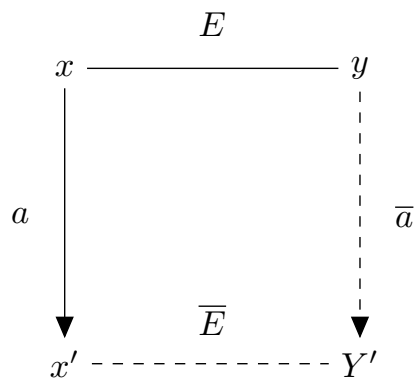

Zag If $y \stackrel{a}{\rightarrow} y^{\prime}$ then there is $X^{\prime} \subseteq E_{\mathcal{A}}$ such that $x \stackrel{\bar{a}}{\rightarrow} X^{\prime}, X^{\prime} \bar{E} y^{\prime}$ and

$$
\operatorname{Pre}(x) \wedge \operatorname{Pre}(y) \models \square_{a}\left(\operatorname{Pre}\left(y^{\prime}\right) \rightarrow \bigvee_{x^{\prime} \in X^{\prime}} \operatorname{Pre}\left(x^{\prime}\right)\right)
$$

In a picture:

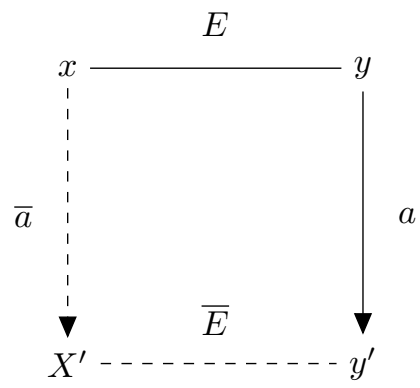

We say that $\mathcal{A}$ and $\mathcal{B}$ emulate, notation $\mathcal{A} \leftrightarrows \mathcal{B}$, if for every $x \in E_{0}^{\mathcal{A}}$ there is $Y \subseteq E_{0}^{\mathcal{B}}$ such that $x \bar{E} Y$ and $\operatorname{Pre}(x) \models \bigvee_{y \in Y} \operatorname{Pre}(y)$, and vice versa.

So every event in one action model is linked to a number of events in the other action model. The precondition of the event in the first model is equivalent to the disjunction of the set of events in the second model. Then if there is a relation to a second world in the first model, for every world in the set there should be a relation to a new set such that the conjunction of the preconditions of the first two worlds implies that any successor that matches the second world in the first action model, also matches one of the worlds in the new set.

This notion of action emulation is sufficient for action model equivalence.

Theorem 9 For any two action models $\mathcal{A}$ and $\mathcal{B}$, if $\mathcal{A} \leftrightarrows \mathcal{B}$ then $\mathcal{A} \equiv \mathcal{B}$ 
Proof. Suppose $\mathcal{A} \leftrightarrows \mathcal{B}$ and let $E$ be an action emulation between $\mathcal{A}$ and $\mathcal{B}$ Let $\mathcal{M}$ be an arbitrary Kripke model. We define a relation $Z$ on $\mathcal{M} \otimes \mathcal{A} \times \mathcal{M} \otimes \mathcal{B}$ as follows:

$$
(w, x) Z(v, y) \text { iff } w=v \text { and } x E y .
$$

We will first show that this relation is total on the actual worlds of $\mathcal{M} \otimes \mathcal{A}$ and $\mathcal{M} \otimes \mathcal{B}$. Suppose $(w, x) \in U_{\mathcal{M} \otimes \mathcal{A}}$. Then $x \in E_{0}^{\mathcal{A}}$ so there must be some $Y \subseteq E_{0}^{\mathcal{B}}$ such that $x \bar{E} Y$ and $\operatorname{Pre}(x) \models \bigvee_{y \in Y} \operatorname{Pre}(y)$. Then $\mathcal{M} \models_{w} \bigvee_{y \in Y} \operatorname{Pre}(y)$, so there is some $y \in Y$ such that $\mathcal{M} \models_{w} \operatorname{Pre}(y)$. But then $(w, x) Z(w, y)$. The proof for the other direction is analogous, so we conclude that $Z$ is total.

Next, we will show that $Z$ is a bisimulation. Suppose $(w, x) Z(w, y)$. Then $x E y$. Invariance is satisfied because both $(w, x)$ and $(w, y)$ inherit their valuation from $w$. For zig, suppose $(w, x) \stackrel{a}{\rightarrow}\left(w^{\prime}, x^{\prime}\right)$. Then $x \stackrel{a}{\rightarrow} x^{\prime}$. By the fact that $x E y$ there must be $Y^{\prime} \subseteq E^{\mathcal{B}}$ such that $y \stackrel{\bar{a}}{\rightarrow} Y^{\prime}, x \bar{E} Y^{\prime}$ and

$$
\operatorname{Pre}(x) \wedge \operatorname{Pre}(y) \models \square_{a}\left(\operatorname{Pre}\left(x^{\prime}\right) \rightarrow \bigvee_{y^{\prime} \in Y^{\prime}} \operatorname{Pre}\left(y^{\prime}\right)\right) .
$$

We have that $\mathcal{M} \models_{w} \operatorname{Pre}(x) \wedge \operatorname{Pre}(y)$ and $\mathcal{M} \models_{w^{\prime}} \operatorname{Pre}\left(x^{\prime}\right)$ and this gives $\mathcal{M}={ }_{w^{\prime}} \bigvee_{y^{\prime} \in Y^{\prime}} \operatorname{Pre}\left(y^{\prime}\right)$, so there must be some $y^{\prime} \in Y^{\prime}$ such that $\mathcal{M} \models{ }_{w^{\prime}} \operatorname{Pre}\left(y^{\prime}\right)$. Because $y^{\prime} \in Y^{\prime}$ it holds that $y \stackrel{a}{\rightarrow} y^{\prime}$ and $x^{\prime} E y^{\prime}$ so $(w, y) \stackrel{a}{\rightarrow}\left(w^{\prime}, y^{\prime}\right)$ and $\left(w, x^{\prime}\right) Z\left(w, y^{\prime}\right)$. This shows the satisfaction of zig. The proof for zag is analogous, so we conclude that $\mathcal{M} \otimes \mathcal{A} \leftrightarrow \mathcal{M} \otimes \mathcal{B}$ and, because $\mathcal{M}$ was arbitrary, $\mathcal{A} \equiv \mathcal{B}$.

This result gives one half of a correspondence between action emulation and action model equivalence.

Turning to the other half, we will show that for canonical action models, action emulation is also necessary for action model equivalence.

Theorem 10 If $\mathcal{A}$ and $\mathcal{B}$ are canonical and $\mathcal{A} \equiv \mathcal{B}$ then $\mathcal{A} \leftrightarrows \mathcal{B}$

Proof. Suppose $\mathcal{A}$ and $\mathcal{B}$ are canonical and $\mathcal{A} \equiv \mathcal{B}$. Let $\mathcal{M}$ be the canonical Kripke model over $\Lambda_{\mathcal{A}} \cup \Lambda_{\mathcal{B}}$. Since $\mathcal{A} \equiv \mathcal{B}$ there is a bisimulation $Z$ between $\mathcal{M} \otimes \mathcal{A}$ and $\mathcal{M} \otimes \mathcal{B}$. Because every world in a canonical Kripke model has a unique valuation, $(w, x) Z(v, y)$ implies $w=v$. Define a relation $E: E_{\mathcal{A}} \times E_{\mathcal{B}}$ as follows:

$$
x E y \text { iff } \exists w \in W_{\mathcal{M}}:(w, x) Z(w, y) .
$$

We will show that $E$ is an action emulation. Suppose $x E y$ and $(w, x) Z(w, y)$. We know that $\operatorname{Pre}(x) \wedge \operatorname{Pre}(y)$ is consistent because $\mathcal{M} \models_{w} \operatorname{Pre}(x) \wedge \operatorname{Pre}(y)$. Suppose $x \stackrel{a}{\rightarrow} x^{\prime}$.

We need to show that there is a set $Y^{\prime}$ such that $y \stackrel{\bar{a}}{\rightarrow} Y^{\prime}, x^{\prime} \bar{E} Y^{\prime}$ and $\operatorname{Pre}(x) \wedge \operatorname{Pre}(y) \models \square_{a}\left(\operatorname{Pre}\left(x^{\prime}\right) \rightarrow \bigvee_{y^{\prime} \in Y^{\prime}} \operatorname{Pre}\left(y^{\prime}\right)\right)$. Let

$$
\begin{aligned}
Y^{\prime}:=\left\{y^{\prime} \in E_{\mathcal{B}} \mid \exists w^{\prime} \in W_{\mathcal{M}}:\right. & (w, x) \stackrel{a}{\rightarrow}\left(w^{\prime}, x^{\prime}\right), \\
& (w, y) \stackrel{a}{\rightarrow}\left(w^{\prime}, y^{\prime}\right), \\
& \left.\left(w^{\prime}, x^{\prime}\right) Z\left(w^{\prime}, y^{\prime}\right)\right\} .
\end{aligned}
$$

It follows from the definition of $Y^{\prime}$ that $y \stackrel{\bar{a}}{\rightarrow} Y^{\prime}$ and $x^{\prime} \bar{E} Y^{\prime}$. 
Now we need to show that $\operatorname{Pre}(x) \wedge \operatorname{Pre}(y) \models \square_{a}\left(\operatorname{Pre}\left(x^{\prime}\right) \rightarrow \bigvee_{y^{\prime} \in Y^{\prime}} \operatorname{Pre}\left(y^{\prime}\right)\right)$ Suppose there is some model $\mathcal{N}$ and worlds $v, v^{\prime} \in W_{\mathcal{N}}$ such that $\mathcal{N} \models{ }_{v} \operatorname{Pre}(x) \wedge$ $\operatorname{Pre}(y), v \stackrel{a}{\rightarrow} v^{\prime}$ and $\mathcal{N} \models{ }_{v^{\prime}} \operatorname{Pre}\left(x^{\prime}\right)$. Let $w^{\prime}:=\bigcup\left\{\varphi \in C\left(\Lambda_{\mathcal{A}} \cup \Lambda_{\mathcal{B}}\right) \mid \mathcal{N} \models{ }_{v^{\prime}} \varphi\right\}$. Then $w^{\prime} \in W_{\mathcal{M}}$ and $\operatorname{Pre}(x) \wedge \operatorname{Pre}(y) \wedge \nabla_{a} \wedge w^{\prime}$ is consistent. Note that $w$ is an atom over $C\left(\Lambda_{\mathcal{A}} \cup \Lambda_{\mathcal{B}}\right)$, and $\operatorname{Pre}(x)$ and $\operatorname{Pre}(y)$ are conjunctions of atoms over $\Lambda_{\mathcal{A}}$ and $\Lambda_{\mathcal{B}}$, respectively. Therefore, $\mathcal{M} \models_{w} \operatorname{Pre}(x) \wedge \operatorname{Pre}(y)$ implies that $\operatorname{Pre}(x) \wedge$ $\operatorname{Pre}(y) \vdash \varphi$ for any $\varphi \in w$. So $w \wedge \diamond_{a} w^{\prime}$ is consistent, and because $\mathcal{M}$ is canonical, $w \stackrel{a}{\rightarrow} w^{\prime}$. Since Pre $\left(x^{\prime}\right) \in w^{\prime}$ then $(w, x) \stackrel{a}{\rightarrow}\left(w^{\prime}, x^{\prime}\right)$. Since $(w, x) Z(w, y)$ then there must be $y^{\prime}$ such that $(w, y) \stackrel{a}{\rightarrow}\left(w^{\prime}, y^{\prime}\right)$ and $\left(w^{\prime}, x^{\prime}\right) Z\left(w^{\prime}, y^{\prime}\right)$. Then $y^{\prime} \in Y^{\prime}$ and $\operatorname{Pre}\left(y^{\prime}\right) \in w^{\prime}$, so $\mathcal{N} \models_{v^{\prime}} \operatorname{Pre}\left(y^{\prime}\right)$ and $\mathcal{N} \models_{v^{\prime}} \bigvee_{y^{\prime} \in Y^{\prime}} \operatorname{Pre}\left(y^{\prime}\right)$. We conclude that $\operatorname{Pre}(x) \wedge \operatorname{Pre}(y) \models \square_{a}\left(\operatorname{Pre}\left(x^{\prime}\right) \rightarrow \bigvee_{y^{\prime} \in Y^{\prime}} \operatorname{Pre}\left(y^{\prime}\right)\right)$. The proof for Zag is analogous. This shows that $E$ is an action emulation.

To see that $E$ is total on the actual events of $\mathcal{A}$ and $\mathcal{B}$, suppose $x \in E_{0}^{\mathcal{A}}$. Let $W_{x}=\left\{w \in W_{\mathcal{M}} \mid \mathcal{M} \models{ }_{w} \operatorname{Pre}(x)\right\}$. By totality of $Z$ and the fact that $(w, x) Z(v, y)$ implies $w=v$ we have that for every $w \in W$ there is an $y$ such that $(w, x) Z(w, y)$. Let $Y=\left\{y \in E_{\mathcal{B}} \mid \exists w \in W_{x}:(w, x) Z(w, y)\right\}$. Then $x \bar{E} Y$ and

$$
\begin{array}{ll}
\operatorname{Pre}(x) & \models \bigvee_{w \in W} w \text { and } \\
\bigvee_{w \in W} w & \models \bigvee_{y \in Y} \operatorname{Pre}(y), \text { so } \\
\operatorname{Pre}(x) & \models \bigvee_{y \in Y} \operatorname{Pre}(y) .
\end{array}
$$

The proof for totality in the other direction is analogous. This shows that $\mathcal{A} \leftrightarrows \mathcal{B}$

Together this gives:

Theorem 11 For any two canonical action models $\mathcal{A}$ and $\mathcal{B}$,

$$
\mathcal{A} \equiv \mathcal{B} \text { iff } \mathcal{A} \leftrightarrows \mathcal{B}
$$

So for canonical action models, non-parametrized action emulation characterizes action model equivalence. This gives a procedure to check whether any two action models are equivalent: just compute the corresponding canonical action models and check whether there is an emulation between them. This is less work than computing the canonical Kripke model as is necessary for checking the existence of a parametrized action emulation, since not all atoms are represented in the canonical action model. Sometimes it may not even be necessary to compute the canonical action model: We have shown that action emulation is sufficient for action equivalence in the general case. So if there is already an action emulation between two non-canonical action models, there is no need to compute the corresponding canonical action models.

\section{Comparison to Propositional Action Emula- tion}

In this section, we will compare our notion of action emulation to the notion of propositional action emulation presented in [4]. It is shown there that propositional action emulation corresponds to action model equivalence for a restricted class of action models, namely the propositional action models. 
Definition 15 An action model is propositional if all preconditions of its events are formulas of classical propositional logic.

Unlike the class of canonical action models, this is a proper subclass of the class of all action models modulo action model equivalence. In other words, it is not possible to find for every non-propositional action model an equivalent propositional one.

Example 12 Consider the following action model:

$$
\mathcal{A}: \quad x^{\mathcal{A}}: \diamond_{a} p
$$

This action model selects all worlds that have an $a$-successor that satisfies $p$. There is no way to construct an equivalent action model that has only propositional preconditions, for propositional preconditions cannot express constraints on the successors of worlds. There is also no way to use relations between action model events to construct an equivalent action model: the only thing the relations between events do is select the relations that will be present in the resulting Kripke model. They do not test the relations in the original Kripke model.

This example shows that the class of propositional action models is indeed a proper subclass of the class of all action models.

Here is the definition of propositional action emulation from [4].

Definition 16 Given two action models $\mathcal{A}$ and $\mathcal{B}$, a relation $E_{P}: E^{\mathcal{A}} \times E^{\mathcal{B}}$ is a propositional action emulation if for any $x \in E^{\mathcal{A}}, y \in E^{\mathcal{B}}$ such that $x E_{P} y$ the following hold:

Consistency $\operatorname{Pre}(x) \wedge \operatorname{Pre}(y)$ is consistent.

Zig If $x \stackrel{a}{\rightarrow} x^{\prime}$ then there is a non-empty set $Y^{\prime} \subseteq E_{\mathcal{B}}$ such that $y \stackrel{\bar{a}}{\rightarrow} Y^{\prime}, x^{\prime} \bar{E}_{P} Y^{\prime}$ and

$$
\operatorname{Pre}\left(x^{\prime}\right) \models \bigvee_{y^{\prime} \in Y^{\prime}} \operatorname{Pre}\left(y^{\prime}\right) .
$$

Zag If $y \stackrel{a}{\rightarrow} y^{\prime}$ then there is a non-empty set $X^{\prime} \subseteq E_{\mathcal{A}}$ such that $x \stackrel{\bar{a}}{\rightarrow} X^{\prime}$, $X^{\prime} \bar{E}_{P} y^{\prime}$ and

$$
\operatorname{Pre}\left(y^{\prime}\right) \models \bigvee_{x^{\prime} \in X^{\prime}} \operatorname{Pre}\left(x^{\prime}\right) .
$$

We say that $\mathcal{A}$ and $\mathcal{B}$ propositionally emulate, notation $\mathcal{A} \leftrightarrows_{P} \mathcal{B}$, if for every $x \in E_{0}^{\mathcal{A}}$ there is $Y \subseteq E_{0}^{\mathcal{B}}$ such that $x \bar{E}_{P} Y$ and $\operatorname{Pre}(x) \models \bigvee_{y \in Y} \operatorname{Pre}(y)$, and vice versa.

It is shown in [4] that for propositional action models, propositional action emulation corresponds to action model equivalence.

Theorem 13 For propositional action models $\mathcal{A}$ and $\mathcal{B}$,

$$
\mathcal{A} \equiv \mathcal{B} \text { iff } \mathcal{A} \leftrightarrows_{P} \mathcal{B}
$$

Also, propositional action emulation is sufficient for action model equivalence, not only for propositional action models, but for action models in general:

Theorem 14 For any action models $\mathcal{A}$ and $\mathcal{B}$,

$$
\mathcal{A} \leftrightarrows_{P} \mathcal{B} \text { implies } A \equiv \mathcal{B}
$$


Proof. Let $\mathcal{A}, \mathcal{B}$ be action models, and suppose $\mathcal{A} \leftrightarrows{ }_{P} B$. Let $E$ be a propositional action emulation between $\mathcal{A}$ and $\mathcal{B}$, such that for every $x \in E_{0}^{\mathcal{A}}$ there is $Y \subseteq E_{0}^{\mathcal{B}}$ such that $x \bar{E}_{P} Y$ and $\operatorname{Pre}(x) \models \bigvee_{y \in Y} \operatorname{Pre}(y)$, and vice versa.

Let $\mathcal{M}$ be an arbitrary Kripke model. Let $C$ be the relation between the worlds of $\mathcal{M} \otimes \mathcal{A}$ and $\mathcal{M} \otimes \mathcal{B}$ given by $(w, d) C(v, e)$ iff $w=v$ and $d E e$.

We show that $C$ is a bisimulation. Let $(w, d) C(v, e)$. Then $w=v$, and invariance holds by the fact the pairs in the update results inherit their valuation from their first component.

Now assume $(w, d) \stackrel{a}{\rightarrow}\left(w^{\prime}, d^{\prime}\right)$. Then $w \stackrel{a}{\rightarrow} w^{\prime}$ and $d \stackrel{a}{\rightarrow} d^{\prime}$, and we get from $d \stackrel{a}{\rightarrow} d^{\prime}$ plus $d E e$ that there is a nonempty set $Y$ of events in $\mathcal{B}$ with $\operatorname{Pre}\left(d^{\prime}\right) \models \bigvee_{y \in Y} \operatorname{Pre}(y)$ and $d^{\prime} E y$ and $e \stackrel{a}{\rightarrow} y$ for each $y \in Y$.

It follows from $\operatorname{Pre}\left(d^{\prime}\right) \models \bigvee_{y \in Y} \operatorname{Pre}(y)$ and $\left.\mathcal{M}\right|_{w^{\prime}} \operatorname{Pre}\left(d^{\prime}\right)$ that there is some $y \in Y$ with $\mathcal{M}={ }_{w^{\prime}} \operatorname{Pre}(y)$. Thus, $\left(w^{\prime}, y\right)$ is a world in $\mathcal{M} \otimes \mathcal{B}$. Since $d^{\prime} E y$ this establishes $(w, e) \stackrel{a}{\rightarrow}\left(w^{\prime}, y\right)$ and $\left(w^{\prime}, d^{\prime}\right) C\left(w^{\prime}, y\right)$. The proof of Zag is analogous.

Can we turn this around? Does equivalence of (canonical) action models imply the existence of a propositional action emulation between them? It turns out it does not. The main difference between propositional action emulation and non-parametrized action emulation as in Definition 14 is in the Zig and Zag conditions, more specifically in the constraint on the preconditions of the events in the sets $X^{\prime}$ and $Y^{\prime}$. For propositional action emulation, the constraint for the Zig case is:

$$
\operatorname{Pre}\left(x^{\prime}\right) \models \bigvee_{y^{\prime} \in Y^{\prime}} \operatorname{Pre}\left(y^{\prime}\right) .
$$

So every world that matches $y^{\prime}$ should also match one of the events in $X^{\prime}$. This condition assures that whenever a world is matched by a successor $x^{\prime}$ of $x$ then it is also matched by a successor in $Y^{\prime}$ of $y^{\prime}$. However, this condition also constrains worlds that match $x^{\prime}$ but are not a successor of a world that matches $x$. We will show now that this condition is too strong.

Definition 14 uses a weaker condition:

$$
\operatorname{Pre}(x) \wedge \operatorname{Pre}(y) \models \square_{a}\left(\operatorname{Pre}\left(x^{\prime}\right) \rightarrow \bigvee_{y^{\prime} \in Y^{\prime}} \operatorname{Pre}\left(y^{\prime}\right)\right)
$$

This condition says that if a world matches $x$ and $y$ then all its successors that match $x^{\prime}$ match one of the worlds in $Y^{\prime}$. This way it only constrains the worlds that are successors of worlds that match both $x$ and $y$. This more subtle condition says exactly what is needed to define action equivalence between canonical models. The fact that the first condition is too strong is shown by the following example.

Example 15 Consider the following two action models: 

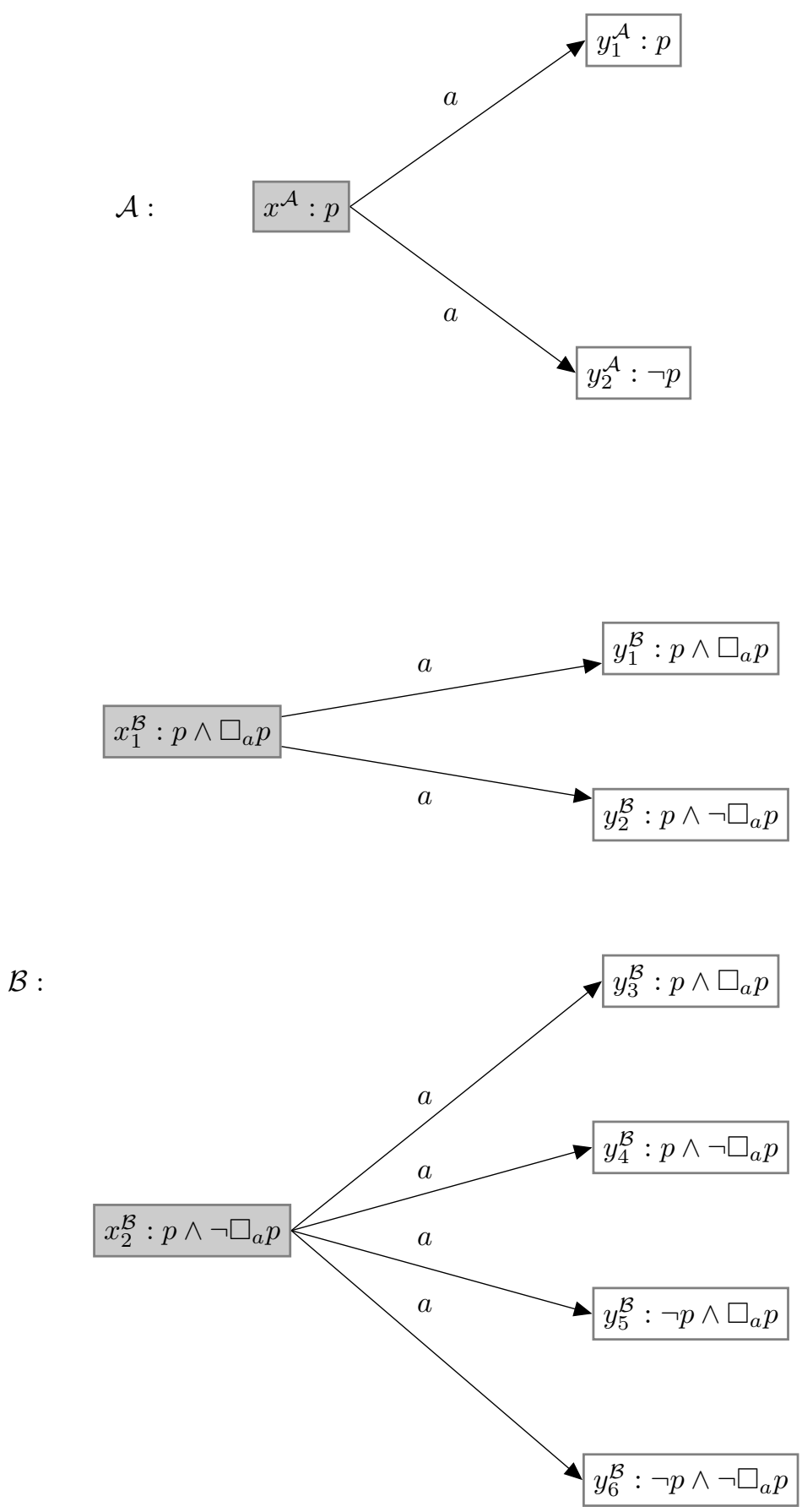

These action models are canonical and equivalent, but they do not propositionally emulate.

To see that these models are equivalent, suppose that some world $w$ matches the event $x^{\mathcal{A}}$ in the first model $\mathcal{A}$. If $w$ satisfies $\square_{a} p$, then it will match $x_{1}^{\mathcal{B}}$ in $\mathcal{B}$ and otherwise it will match $x_{2}^{\mathcal{B}}$ in $\mathcal{B}$. Suppose $w$ has some successor that matches $y_{1}^{\mathcal{A}}$. Then this successor satisfies $p$ so it will match either $y_{1}^{\mathcal{B}}$ or $y_{2}^{\mathcal{B}}$ if $w$ 
matched $x_{1}^{\mathcal{B}}$, or $y_{3}^{\mathcal{B}}$ or $y_{4}^{\mathcal{B}}$ if $w$ matched $x_{2}^{\mathcal{B}}$. Suppose $w$ has some successor that matches $y_{2}^{\mathcal{A}}$. Then this successor does not satisfy $p$, so $w$ does not satisfy $\square_{a} p$, so $w$ matched $x_{2}^{\mathcal{B}}$. In this case the successor of $w$ will match $y_{5}^{\mathcal{B}}$ or $y_{6}^{\mathcal{B}}$.

Another way to see that these canonical models are equivalent is by checking that the relation given by

$E=\left\{\left(x^{\mathcal{A}}, x_{1}^{\mathcal{B}}\right),\left(y_{1}^{\mathcal{A}}, y_{1}^{\mathcal{B}}\right),\left(y_{1}^{\mathcal{A}}, y_{2}^{\mathcal{B}}\right),\left(x^{\mathcal{A}}, x_{2}^{\mathcal{B}}\right),\left(y_{1}^{\mathcal{A}}, y_{3}^{\mathcal{B}}\right),\left(y_{1}^{\mathcal{A}}, y_{4}^{\mathcal{B}}\right),\left(y_{2}^{\mathcal{A}}, y_{5}^{\mathcal{B}}\right),\left(y_{2}^{\mathcal{A}}, y_{6}^{\mathcal{B}}\right)\right\}$

is an action emulation between $\mathcal{A}$ and $\mathcal{B}$.

To see that the models do not propositionally emulate, observe that $x_{1}^{\mathcal{B}}$ does not emulate with $x^{\mathcal{A}}$ (or any other event in $\mathcal{A}$ ). Because from $x^{\mathcal{A}}$ there is a relation to $y_{2}^{\mathcal{A}}$, while there is no set of successors of $x_{1}^{\mathcal{B}}$ such that the precondition $\neg p$ implies the disjunction of preconditions of events in this set.

This shows that propositional action emulation does not characterize action equivalence between canonical action models, nor action model equivalence between action models in general.

\section{Conclusion}

In this paper we studied the properties of action models. Action models are applied to Kripke models and they are equivalent if they give equivalent results for all possible Kripke models. Our aim was to find a relation between action models that signifies when they are equivalent, just like bisimulation does for Kripke models.

Finding an appropriate relation that signifies equivalence of action models is complicated by the fact that multiple worlds in the Kripke model may match one world in the action model, and vice versa. We circumvent this complication by considering canonical action models. Our main result is a new notion of (non-parametrized) action emulation that is necessary and sufficient for action model equivalence for canonical action models. Because every action model has an equivalent canonical action model this gives a method to determine whether any two action models are equivalent.

Since our new notion of action emulation is sufficient for actiom model equivalence, even if the action models are not canonical, and since the notion of propositional action emulation from [4] is sufficient for action model equivalence, we can propose the following procedure for finding out if two action models are equivalent.

- First try to find a propositional action emulation between the models. If this works, the models are equivalent.

- If this does not work, try to find a (non-parametrized) action emulation between the models. If this works, the models are equivalent.

- If this does not work either, check whether there is a (non-parametrized) action emulation between the canonical models. This gives a conclusive answer.

We leave for future research whether our notion of action emulation is equivalent to action model equivalence on the full class of all action models, not just the canonical ones. 
We compared our notion of action emulation to two notions given in [4]: that of parametrized action emulation and that of propositional action emulation. Our notion of action emulation has a clear advantage to parametrized action emulation: there is no need to compute a separate relation for every world in the canonical Kripke model. The advantage compared to propositional action emulation is that on the class of canonical action models, propositional action emulation can only show equivalence, not non-equivalence. Our method using non-parametrized action emulation works for all canonical action models. Because every action model has an equivalent canonical action model, this gives a solution for the entire class of action models.

\section{References}

[1] Alexandru Baltag, Lawrence S. Moss, and Slawomir Solecki. The logic of public announcements, common knowledge, and private suspicions. In Proceedings of the 7th conference on Theoretical Aspects of Rationality and Knowledge (TARK' '98), pages 43-56, 1998.

[2] Patrick Blackburn, Maarten de Rijke, and Yde Venema. Modal Logic. Cambridge University Press, 2001.

[3] Floor Sietsma. Logics of Communication and Knowledge. PhD thesis, University of Amsterdam, 2012.

[4] Jan van Eijck, Ji Ruan, and Tomasz Sadzik. Action emulation. Synthese, 185(1):131-151, 2012. 